\title{
Modeling of the archery bow and arrow vibrations
}

\author{
I. Zaniewski* \\ Casimir Pulaski Technical University, Radom, Poland
}

Received 28 April 2005

Revised 27 September 2007

\begin{abstract}
Vibration processes in the compound and open kinematical chain with an external link, as a model of an archery bow and arrow system, are evaluated. A mechanical and mathematical model of bend oscillations of the system during accelerate motion of the external link is proposed. Correlation between longitudinal acceleration and natural frequencies is obtained. There are recommendations regarding determination of virtual forms to study arrow vibrations and buckling. The models and methods have been adapted for realization into the engineering method using well-known mathematical software packages.
\end{abstract}

Keywords: Bow, kinematical chain, vibration, buckling, natural forms, frequencies

\section{Introduction}

The sports which are played with mechanical devices represent a large proportion of both human competitive and recreational activity. However, the level of our knowledge and understanding of technique in these activities appears to lag behind those of other popular sporting activities. There are some obvious reasons for this discrepancy; one of them is an insufficient level of mechanical and mathematical modeling and computer simulation in this field. Sport archery is a good model for the study of the mechanical processes in the 'Man and Device' system [14]. Archery is a sport for people of all ages, and is challenging whether practiced alone or as part of a group, as the competition is between the archer and their device (bow and arrows). Archery is a mass participation recreational sport. Thus, up-to-date equipment is both required and desirable [1].

A typical bow is set-up so that anyone can shoot it reasonably well; therefore it will not perform optimally for each individual archer. However, by making adjustments in the bow set-up, it is possible to significantly improve an archer's performance, sometimes dramatically. The process of adjustment of the bow set-up is called tuning. A bow which has been correctly tuned to the individual user, will aid them to perform a shot at the peak of their ability. A bow that is fine-tuned for one archer will not, in most cases be the optimum set-up for any other archer [3].

A modern sport bow is suited for shooting vertically within its main plane. Vertical adjustment can be defined, as providing an optimal combination of bow parameters to obtain a zero angle of attack of an arrow. It is a significant part of preparation for shooting, which an archer has to manage with a bow. With a zero angle of attack, an arrow has the lowest aerodynamic drag and therefore the greatest possible speed. Higher velocity results in better conditions for good sport performance, because any angle of attack causes the arrow to wobble in the vertical plane [15].

The aim of this research is to model and study vibration processes and instability in the bow and arrow system.

*Address for correspondence: Malczewskiego 22, Radom 26-600, Poland. Tel.: +48 483617886 - day; +48 483618809 - night; Fax: +48 48 3617803; E-mail: igor_zaniewski@ukr.net. 


\section{Overview on the archery bow modeling}

A common scheme of the modern sport bow had been designed in the first quarter of the last century. The main features of it are a rigid central part named a riser (or a handle) and two flexible limbs mounted on the ends of the handle. The unstrung limbs have curved horns at the free ends, which are bent backward of the internal part of the bow [1].

There are two experimental methods (Bare Shaft Method developed by Max Hamilton and Eliason Method) for tuning a bow in the vertical plane [1]. These methods are based on laborious procedures through a lengthy and complicated trial and error phase; they entail considerable effort and much time. Furthermore, these methods allow only the variation of one single parameter - the nocking height of an arrow. Another variable which is believed to be important and has not been taken into consideration in the frames of the methods, is the initial set-up angles of the limbs relative to the handle.

Bow and arrow common vibrations were quantified using high-speed video footage [12]. There are primary theoretical studies on modeling the vibrations of the archery bow and arrow system in the lateral plane $[4,9,19]$, but as yet no research has been undertaken on modeling the system in the main plane.

Hickman and Klopsteg [3] initiated scientific research in the field of sport archery. They investigated geometrical parameters of bows using experimental methods to investigate the limbs and string shapes over a static force. Hickman created the first mechanical and mathematical model of sport bow deformations. Instead of flexible limbs, he introduced two straight rigid beams jointed to a handle with hinges. Limb stiffness was modeled with a spiral spring placed around a hinge's axis. An arrow was modeled as a particle placed on the axis of symmetry of the system. A third of the string's mass was jointed to the mass of the arrow; the remaining mass was jointed to that of the limb tips. Hickman designed Lagrange function of one degree of freedom and an equation of motion. Marlow [7] improved the model taking into consideration the string's elasticity. Hence, he got the mathematical model as Lagrange function of two degrees of freedom and corresponding equations.

The next step towards improving the model was to model the limbs as thin flexible beams, applying a finitedifference technique discretization to solve the static problem [5,10]. B.M. Kooi [6,11] added to the model equations describing a contact process of a string and a curved part of limb in outlining area of it. Using the partial differential equations and a finite-difference technique discretizating both spatial and temporal variables he solved the dynamic problem.

All the mentioned models were created for a symmetric scheme of a bow. A number of studies have been concerned with creating an asymmetrical model of a sport bow, to study the problem of internal arrow ballistics [13, 16-20]. The model exists in two versions; i) a relatively simple model, based on the Hickman's model and the arrow is modeled as a rigid shaft [3] and ii) a more advanced model which uses the Kooi's model and the arrow is modeled as a flexible shaft with particles at the ends [4]. The simpler of the two models shows all the essential properties regarding the problem of bow tuning in the vertical plane. The more advanced model provides some additional accuracy. The simple model gives a much clearer understanding of the aim of the study, mechanics and results of the tuning. Therefore, the simpler model is used in this paper.

The first attempt at the problem of the adjustment of the asymmetric sport bow has been made using a static model of the bow and arrow system by Gros and Zanevskyy [2]. Although the results of modeling the frame using the quasi-static approach have some practical value, they should be considered as an approximation. Because a dynamic character of the bow and arrow common motion, we have to study a dynamic problem. For example, arrow acceleration is about 600-700 gravity units. There is a dynamic analysis of the traditional asymmetric Japan bow [8] but this specific bow is very different in comparison the modern sport bow.

Our mechanical model of the bow and arrow system is based on the compound kinematical chain with a free member (an arrow) that has only one kinematical pair, i.e. with the string. The hinge unites together the arrow and two branches of the string; this is the determining feature of the compound chain.

Bow and arrow common motion is characterized by an intensive dynamic process, i.e. impulse buckle and bend vibrations. The lateral part of the spatial motion can be eliminated because it's a negligible portion of the total displacement and energy of the system. The inertial and elastic properties of the bow are taken into account with a simple mechanical oscillator united at the arrow tail [20]. 


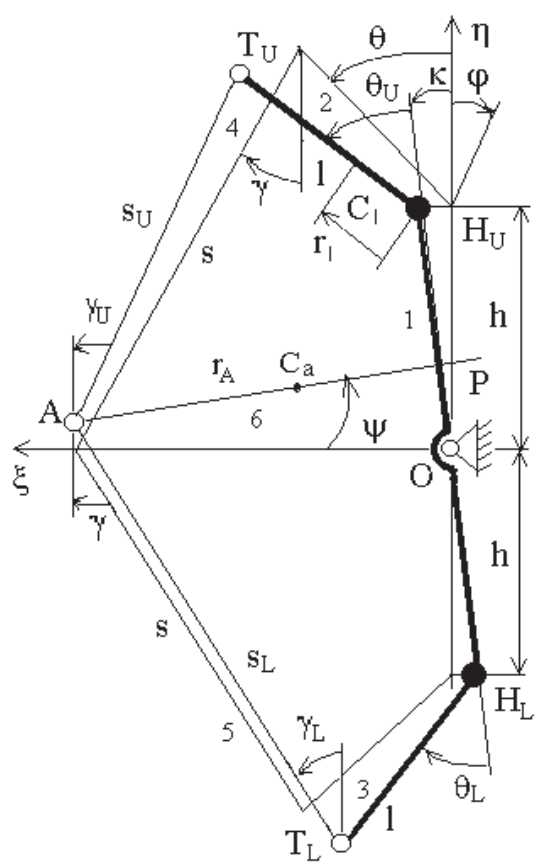

Fig. 1. Symmetric scheme of the bow and arrow system.

An adequate mathematical model is presented as an ordinary differential equation with non-constant coefficients. The numerical solution of the problem was created using an iterative process with approximation of natural modes as a polynomial series [19].

Bow and arrow common motion was studied using a model of a kinematical chain with non-elastic shift-members. Here, a mathematical model was presented as a system of partial differential equations with non-constant coefficients with respect to both time and the longitudinal coordinate as well as the initial conditions. The solution of the adequate Cauchy problem was obtained with Runge-Kutta method.

The mechanical and mathematical model of arrow buckle and longitudinal bend vibration can be reduced to a system of ordinary differential equations by substituting virtual functions, which are similar to the natural modes and meet all the boundary conditions (or a part of them). Application of this form of energetic method makes possible to get a solution with precision that is acceptable for engineering calculations. The objective criterion of successful 'construction' of the hypothetical forms is a difference between rated and natural values of frequencies $[16,18]$.

\section{Symmetrical scheme}

The asymmetry of the system in its main plane is small (3-4\%), therefore by taking into account a common precision that is acceptable for engineering calculations, it is possible to use in modeling hypothetic forms from the symmetrical scheme of the chain (Appendix). A geometrical linear model of the Euler-Bernoulli beam was considered to be acceptable because; i) an arrow length is $\sim 100$ time bigger than the diameter of its aluminum alloy cylinder tube and ii) bend and buckle deformation amplitude was concluded to be in the range of $5 \%$ of the arrow length using high speed video footage [12].

Geometric and force equations for the neutral position of the chain are (Fig. 1):

$$
h+l \cos \theta=s \cos \gamma ; \quad l \sin \theta+s \sin \gamma=\xi_{A} ; \quad c_{s}\left(s-s_{0}\right) l \sin (\theta+\gamma)=c_{l}(\theta+\varphi),
$$

where $c_{l}$ is a virtual stiffness of a limb located in the hinge at the end of a riser; $c_{s}$ is a stiffness of a string branch; $s_{0}$ is a half-length of the string; $\varphi$ is an angle of the virtual limb at the free position (i.e. without a string); $\xi O \eta$ is 
'fixed' to the archer hand immovable system of coordinates. The sense of the rest symbols is clear from the scheme (see Fig. 1).

For a small displacement from the neutral position, related equations regarding the upper $(\mathrm{U})$ and lower $(\mathrm{L})$ parts of the kinematical chain are:

$$
\begin{aligned}
& h+l \cos \left(\theta_{U / L} \pm \kappa\right)=s_{U / L} \cos \gamma_{U / L} \pm \eta_{A} ; \quad \pm h \kappa+l \sin \left(\theta_{U / L} \pm \kappa\right)+s_{U / L} \sin \gamma_{U / L}=\xi_{A} ; \\
& c_{s}\left(s_{U / L}-s_{0}\right) l \sin \left(\theta_{U / L}+\gamma_{U / L}\right)=c_{l}\left(\theta_{U / L}+\varphi\right),
\end{aligned}
$$

where $\kappa$ is the angular displacement of the riser. We mark in the twin subdivides (U/L) the upper letter concerns the upper part of the chain and opposite.

The geometric equations for the neutral Eq. (1) and displaced Eq. (2) positions of the chain are combined together to form:

$$
\theta_{U / L}=\theta+\Delta \theta_{U / L} ; \quad \gamma_{U / L}=\gamma+\Delta \gamma_{U / L} ; s_{U / L}=s+\Delta s_{U / L}
$$

where the letter $\Delta$ marks small displacements and deformations. After substituting Eq. (3) into Eq. (2) and transformations using Eq. (1), we get a system of three linear equations $\left(\Delta \theta_{U}+\Delta \theta_{L}\right),\left(\Delta \gamma_{U}+\Delta \gamma_{L}\right),\left(\Delta s_{U}+\Delta s_{L}\right)$ homogeneous algebraic equations:

$$
\begin{aligned}
& l\left(\Delta \theta_{U}+\Delta \theta_{L}\right) \cos \theta+s\left(\Delta \gamma_{U}+\Delta \gamma_{L}\right) \cos \gamma+\left(\Delta s_{U}+\Delta s_{L}\right) \sin \gamma=0 ; \\
& l\left(\Delta \theta_{U}+\Delta \theta_{L}\right) \sin \theta-s\left(\Delta \gamma_{U}+\Delta \gamma_{L}\right) \sin \gamma+\left(\Delta s_{U}+\Delta s_{L}\right) \cos \gamma=0 ; \\
& {\left[c_{s}\left(s-s_{0}\right) l \cos (\theta+\gamma)-c_{l}\right]\left(\Delta \theta_{U}+\Delta \theta_{L}\right)+c_{s}\left(s-s_{0}\right) l \cos (\theta+\gamma)\left(\Delta \gamma_{U}+\Delta \gamma_{L}\right)} \\
& \quad+c_{s} l \sin (\theta+\gamma)\left(\Delta s_{U}+\Delta s_{L}\right)=0 .
\end{aligned}
$$

The determinant of the system as for real dimensions of the target sport archery bows [1].

$\frac{s_{0}}{l}<2 ; \frac{s-s_{0}}{s_{0}}<0,02 ; 20^{\circ}<\theta+\gamma<180^{\circ}$, which are designed in the frame of FITA (International Archery Federation: www.archery.org) Standard is not equal zero:

$$
c_{s}\left(s-s_{0}\right) l^{2} \cos ^{2}(\theta+\gamma)+c_{l} s+c_{s} l^{2} s \sin ^{2}(\theta+\gamma) \times\left[1-\frac{s_{0}}{l} \frac{s-s_{0}}{s_{0}} \frac{\cos (\theta+\gamma)}{\sin ^{2}(\theta+\gamma)}\right]>0 .
$$

Because the last unequal, the system of Eq. (4) has only zero solutions:

$$
\Delta \theta_{U}+\Delta \theta_{L}=0 ; \quad \Delta \gamma_{U}+\Delta \gamma_{L}=0 ; \quad \Delta s_{U}+\Delta s_{L}=0 .
$$

So, we can reduce the model and study small vibrations of the symmetrical chain using the following equations:

$$
\Delta \theta_{U} \equiv-\Delta \theta_{L}=\tau ; \quad \Delta \gamma_{U} \equiv-\Delta \gamma_{L} ; \quad \Delta s_{U} \equiv-\Delta s_{L} ; \quad \Delta \theta_{U}^{\prime} \equiv-\Delta \theta_{L}^{\prime}=\tau^{\prime} .
$$

As a mathematical model of the vibration, we use a system of Lagrange equations of the second order:

$$
\frac{d}{d t}\left(\frac{\partial T}{\partial q_{i}^{\prime}}\right)-\frac{\partial T}{\partial q_{i}}+\frac{\partial P}{\partial q_{i}}=0
$$

where $T$ and $P$ are consequently the kinetic and potential energy of the system, $q_{i}$ are generalized coordinates; $t$ is a time; prefix shows a partial derivation in time, i.e. $\left({ }^{\prime}\right) \equiv \partial / \partial t$.

Taking into account (5), expressions of the energy can be written as:

$$
\begin{aligned}
& T=\frac{1}{2}\left\{m_{A} \eta^{\prime} A^{2}+\left(I_{H}+2 m_{l} h^{2}\right) \kappa^{\prime 2}+2 I_{l}\left(\tau^{\prime}+\kappa^{\prime}\right)^{2}+4 m_{l} r_{l} h \kappa^{\prime}\left(\tau^{\prime}+\kappa^{\prime}\right)[\cos \theta-(\tau+\kappa) \sin \theta]\right\} ; \\
& P=P_{l}+P_{s},
\end{aligned}
$$

where $m_{A}=m_{a A}+1 / 3 m_{s}$ is the virtual mass of the arrow with a portion of string mass pinned to the nock point ( $m_{s}$ is the mass of a string); $I_{H}$ is the moment of inertia of a riser, a sign and stabilizes relatively the pivot point $(\mathrm{O}) ; m_{l}$ is the mass of a limb with an additional portion of string mass $\left(1 / 3 m_{s}\right)$ pinned to the tip point; $I_{l}$ is the moment of inertia of a limb relative to its joint to the riser with addition the same part of string mass; $r_{l}$ is the 
distance from the center of mass of the limb to the joint; $P_{l}=\frac{1}{2} c_{l}\left[\left(\theta_{U}+\varphi\right)^{2}+\left(\theta_{L}+\varphi\right)^{2}-2(\theta+\varphi)^{2}\right]=c_{l} \tau^{2}$ is a difference in potential energy of the limb between neutral and asymmetric positions of the chain; $P_{s}=$ $\frac{c_{s}}{2}\left[\left(s_{U}-s_{0}\right)^{2}+\left(s_{L}-s_{0}\right)^{2}-2\left(s-s_{0}\right)^{2}\right]=c_{s} \Delta s^{2}$ is the same difference in potential energy of the string; $\Delta s=-\left[l \tau \sin (\theta+\gamma)+\left(\xi_{A} \kappa+\eta_{A}\right) \cos \gamma\right]$.

Setting generated co-ordinates $q_{i} \equiv \kappa, \tau, \eta_{A}$, we get differential equations of vibrations of the chain in its main plane:

$$
\begin{aligned}
& I_{\kappa} \kappa^{\prime \prime}+I_{\tau} \tau^{\prime \prime}+c_{s} \Delta s \xi_{A} \cos \gamma=0 ;_{\frac{1}{2}} m_{A} \eta_{A}^{\prime \prime}+c_{s} \Delta s \cos \gamma=0 \\
& I_{l} \tau^{\prime \prime}+c_{l} \tau+c_{s} l \Delta s \sin (\theta+\gamma)+I_{\tau} \kappa^{\prime \prime}=0,
\end{aligned}
$$

where $I_{\kappa}=\frac{1}{2} I_{H}+m_{l} h^{2}+I_{l}+2 m_{l} h r_{l} \cos \theta ; I_{\tau}=I_{l}+m h r_{l} \cos \theta$.

Geometrical parameters in the neutral position of the chain $\left(\xi_{A}, \theta, \gamma\right)$ are solutions of the Eq. (1).

We get natural frequencies $\omega$ and natural modes of vibration by substituting solutions in the form: $q_{i}=\alpha_{i} \cos \omega t$ into the Eq. (8), where $\alpha_{i}$ are amplitudes of vibration. The main determinant of the obtained system of linear (relative the amplitudes) equations should be equal to zero:

$$
\left|\begin{array}{lll}
b_{\kappa \kappa} & b_{\kappa \tau} & b_{\kappa \eta} \\
* & b_{\tau \tau} & b_{\tau \eta} \\
* & * & b_{\eta \eta}
\end{array}\right|=0
$$

where

$$
\begin{aligned}
& b_{\kappa \kappa}=c_{s}\left(\xi_{A} \cos \gamma\right)^{2}-\omega^{2} I_{\kappa} ; \quad b_{\kappa \eta}=c_{s} \xi_{A} \cos ^{2} \gamma \\
& b_{\kappa \tau}=c_{s} l \sin (\theta+\gamma) \xi_{A} \cos \gamma-\omega^{2} I_{\tau} ; \\
& b_{\tau \tau}=c_{s}[l \sin (\theta+\gamma)]^{2}+c_{l}-\omega^{2} I_{l} ; \quad b_{\eta \eta}=c_{s} \cos ^{2} \gamma-\frac{1}{2} m_{A} \omega^{2} .
\end{aligned}
$$

We can make a previous analysis of the chain vibration by assuming the arrow to be a rigid shaft. This assumption is based on the arrow's elastic deformations being significantly small in comparison with the virtual displacement which the limbs transferred to the arrow tail A (see Fig. 1) [15]. We also pin inertial properties of the arrow to the tail point A.

We get virtual mass of the arrow from the system of equations - equal linear momentum and zero angular momentum relatively the pinned point $\mathrm{A}$ :

$$
\int_{0}^{l_{a}} \mu(z) \eta_{a}^{\prime}(z) d z=m_{a A} \eta_{A}^{\prime} ; \quad \int_{0}^{l_{a}} \mu(z) \eta_{a}^{\prime}(z) z d z=0
$$

where $\mu(z)$ is the distributed mass of the arrow; $l_{a}$ is its length; $\eta_{a}(z)=\eta_{A}+\psi z$ is the displacement of the arrow axis as a rigid shift. Substituting the last form into Eq. (10), we get the following expression for the virtual mass: $m_{a A}=\frac{m_{a} r_{a}^{2}}{r_{A}^{2}+r_{a}^{2}}$, where $r_{A}=\frac{\int_{0}^{l_{a}} \mu(z) z d z}{m_{a}}$ is a distance between the tail and the center of mass of the arrow; $m_{a}$ is the total mass of the arrow; $r_{a}=\sqrt{\frac{\int_{0}^{l_{a}} \mu(z)\left(z-r_{A}\right)^{2} d z}{m_{a}}}$ is the radius of inertia of the arrow.

Lets examine a modern sport bow and an arrow of mean size (www.hoytusa.com; www.eastonarchery.com): $I_{\kappa}=0.671 \mathrm{kgm}^{2} ; m_{l}=0.0953 \mathrm{~kg} ; h=0.4343 \mathrm{~m} ; I_{l}=0.006344 \mathrm{kgm}^{2} ; r_{l}=0.2165 \mathrm{~m} ; l=0.48 \mathrm{~m} ;$ $c_{l}=120.78 \mathrm{Nm} ; c_{s}=14000 \mathrm{~N} / \mathrm{m} ; s_{0}=0.85 \mathrm{~m} ; \xi_{A}=l_{a}=0.7 \mathrm{~m} ; \theta=0.7608 \mathrm{rad} ; \gamma=0.4410 \mathrm{rad} ;$ $m_{a}=0.0091 \mathrm{~kg}$. Substituting these parameters into Eq. (9), we get values of the natural circular frequencies $\omega_{1}=132,293 \mathrm{~s}^{-1}$ and $\omega_{2}=1730 \mathrm{~s}^{-1}$. If we disregard the elasticity of the string $\left(c_{s}=\infty\right)$, the chain has only one natural frequency $\omega=132,358 \mathrm{c}^{-1}$ that differs of the exact solution for the first natural frequency by approximately $0.05 \%$.

The problem was solved using the MathCAD package. Standard function were applied to calculate the value of the determinant from Eq. (9) changing consequently squad values of frequencies. If the value of the determinant changed its sign, the natural frequency had been met. Using the interpolation, we calculated its value. 


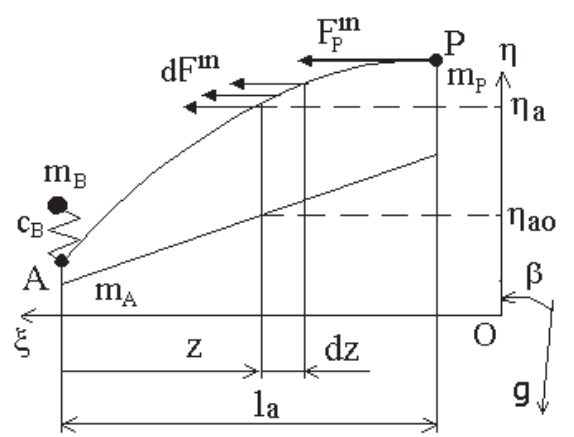

Fig. 2. Virtual scheme of the system: g-gravity direction.

\section{A virtual model of the system}

Bow and arrow common motion is accompanied by strong vibrations and the impulse buckle of the arrow [12]. An engineering method for calculation of the process could be based on the model of longitudinal bend and buckle of a free shift based on the Euler-Bernoulli model with a simple mechanical oscillator connected to its back end [19].

String stiffness is significantly larger than virtual limb stiffness; therefore it is reasonable to assume a linear mechanical scheme of the model (Fig. 2). It is possible to study vibrations of a shift dividing them into natural modes. The main quota of the whole displacement belongs to the first or second natural modes. These modes have one, two or three nodes. Natural modes could be written using Krylov functions, although accuracy of engineering calculations allows the use of hypothetical functions [17]. It's considered reasonable to design hypothetical function as a sum of linear function and harmonics in the form of sinusoid and a half of sinusoid:

$$
\eta_{a 3}=\eta_{A}+\psi z+f_{2} \sin \frac{\pi z}{l_{a}}+f_{3} \sin \frac{2 \pi z}{l_{a}},
$$

where $\psi$ is the attitude angle of the arrow; $f_{2}, f_{3}$ are amplitudes of deformation of the arrow shaft.

Potential and kinetic energy of the system are:

$$
\begin{aligned}
& P=\frac{1}{2} c_{B}\left(\eta_{B}-\eta_{A}\right)^{2}+\frac{1}{2} \int_{0}^{l_{2}} \varepsilon\left(z_{a}\right)\left(\frac{\partial^{2} \eta_{a}}{\partial z_{a}^{2}}\right)^{2} d z_{a}+\frac{1}{2} \xi_{A}^{\prime \prime} \int_{0}^{l_{a}} \mu\left(z_{a}\right)\left[\int_{0}^{z_{a}}\left(\frac{\partial \eta_{a}}{\partial z_{a}}\right)^{2} d \chi+\left(\frac{\partial \eta_{a}}{\partial z_{a}}\right)_{z_{a}=l_{a}}^{2}\right] d z_{a} ; \\
& T=\frac{1}{2} m_{B} \eta^{\prime} B^{2}+\frac{1}{2} \int_{0}^{l_{a}} \mu_{a}\left(z_{a}\right) \eta^{\prime} a^{2} d z_{a},
\end{aligned}
$$

where $c_{B}$ is the virtual stiffness of two limbs; $m_{B}$ is the virtual mass of the bow; $\varepsilon\left(z_{a}\right)$ is the distributed stiffness of the arrow shaft. Substituting expression of the hypothetic form Eq. (11) into the last two expressions of energy, the following expression is obtained for the cylindrical arrow:

$$
\begin{aligned}
& P=\frac{1}{2} c_{B}\left(\eta_{B}-\eta_{A}\right)^{2}+\frac{\pi^{4} \varepsilon}{4 l_{a}^{3}} f_{2}^{2}+\frac{4 \pi^{4} \varepsilon}{l_{a}^{3}} f_{3}^{2} \\
& +\frac{1}{2} \xi_{A}^{\prime \prime}\left[m_{s h}\left(\frac{l_{a}}{2} \psi^{2}+\frac{4}{\pi} \psi f_{2}+\frac{\pi^{2}}{4 l_{a}} f_{2}^{2}+\frac{\pi^{2}}{l_{a}} f_{3}^{2}+\frac{16}{3 l_{a}} f_{2} f_{3}\right)+m_{P}\left(l_{a} \psi^{2}+\frac{\pi^{2}}{2 l_{a}} f_{2}^{2}+\frac{2 \pi^{2}}{l_{a}} f_{3}^{2}\right)\right] ; \\
& T=\frac{1}{2}\left[\begin{array}{l}
m_{s h}\left(\begin{array}{l}
{\eta^{\prime}}_{A}^{2}+\frac{l_{a}^{2}}{3} \psi^{\prime 2}+\frac{f^{\prime 2}}{2}+\frac{f^{\prime 2}}{2}- \\
\frac{l_{a}}{\pi} \psi^{\prime} f^{\prime}{ }_{3}+l_{a} \eta^{\prime}{ }_{A} \psi^{\prime}+\frac{4}{\pi} \eta^{\prime}{ }_{A} f^{\prime}{ }_{2}+\frac{2}{\pi} l_{a} \psi^{\prime} f^{\prime}{ }_{2}
\end{array}\right) \\
+m_{A}{\eta^{\prime 2}}_{A}+m_{P}\left(\eta^{\prime}{ }_{A}+l_{a} \psi^{\prime}\right)^{2}+m_{B}{\eta^{\prime}}_{B}{ }_{B}
\end{array}\right],
\end{aligned}
$$

where $m_{s h}$ is the mass of the arrow shaft, excluding the mass of the tip and tail. 
Setting generated coordinates $q_{i} \equiv \eta_{A}, \eta_{B}, \psi, f_{2}, f_{3}$ and substituting expressions of energy into the Lagrange Eq. (6), the following differential equations of the vibrations of the system are obtained:

$$
\begin{aligned}
& \left(m_{s h}+m_{A}+m_{P}\right) \eta_{A}^{\prime \prime}+\left(\frac{m_{s h}}{2}+m_{P}\right) l_{a} \psi^{\prime \prime}+\frac{2}{\pi} m_{s h} f_{2}^{\prime \prime}-c_{B}\left(\eta_{B}-\eta_{A}\right)=0 ; \\
& m_{B} \eta_{B}^{\prime \prime}+c_{B}\left(\eta_{B}-\eta_{A}\right)=0 ; \\
& \left(\frac{m_{s h}}{3}+m_{P}\right) l_{a} \psi^{\prime \prime}+\left(\frac{m_{s h}}{2}+m_{P}\right) \eta_{A}^{\prime \prime}+\frac{m_{s h}}{\pi} f_{2}^{\prime \prime}-\frac{m_{s h}}{2 \pi} f_{3}^{\prime \prime}+\left[\left(\frac{m_{s h}}{2}+m_{P}\right) l_{a} \psi+\frac{2}{\pi} m_{s h} f_{2}\right] \frac{\xi_{A}^{\prime \prime}}{l_{a}}=0 ; \\
& \frac{m_{s h}}{2} f_{2}^{\prime \prime}+\frac{2}{\pi} m_{s h} \eta_{A}^{\prime \prime}+\frac{m_{s h}}{\pi} l_{a} \psi^{\prime \prime}+\frac{\pi^{4} \varepsilon}{2 l_{a}^{3}} f_{2}+\left[\frac{\pi^{2}}{2}\left(\frac{m_{s h}}{2}+m_{P}\right) f_{2}+\frac{2}{\pi} m_{s h} l_{a} \psi+\frac{8}{3} m_{s h} f_{3}\right] \frac{\xi_{A}^{\prime \prime}}{l_{a}}=0 ; \\
& \frac{m_{s h}}{2} f_{3}^{\prime \prime}-\frac{m_{s h}}{2 \pi} l_{a} \psi^{\prime \prime}+\frac{8 \pi^{4} \varepsilon}{l_{a}^{3}} f_{3}+\left[\pi^{2} m_{s h} f_{3}+\frac{8}{3} m_{s h} f_{2}+2 \pi^{2} m_{P} f_{3}\right] \frac{\xi_{A}^{\prime \prime}}{l_{a}}=0 .
\end{aligned}
$$

Like Eq. (9) the main determinant of the obtained system Eq. (13) of linear relatively the amplitudes equations should be equal zero:

$$
\left|\begin{array}{ccccc}
b_{00} & b_{01} & b_{02} & b_{03} & b_{04} \\
* & b_{11} & b_{12} & b_{13} & b_{14} \\
* & * & b_{22} & b_{23} & b_{24} \\
* & * & * & b_{33} & b_{34} \\
* & * & * & * & b_{44}
\end{array}\right|=0
$$

where

$$
\begin{aligned}
& b_{00}=\nu-\left(1+\varsigma_{A}+\varsigma_{B}\right) \Omega^{2} ; \quad b_{01}=-\nu ; \quad b_{02}=-\left(\frac{1}{2}+\varsigma_{P}\right) \Omega^{2} ; \\
& b_{03}=-\frac{2}{\pi} \Omega^{2} ; \quad b_{04}=b_{12}=b_{13}=b_{14}=0 ; \quad b_{11}=\nu-\varsigma_{B} \Omega^{2} ; \\
& b_{22}=-\left(\frac{1}{3}+\varsigma_{P}\right) \Omega^{2}-\left(\frac{1}{2}+\varsigma_{P}\right) \Phi ; \quad b_{23}=-\frac{\Omega^{2}+2 \Phi}{\pi} ; \quad b_{24}=\frac{\Omega^{2}}{2 \pi} ; \\
& b_{33}=\frac{1}{2}\left\{\pi^{2}\left[4 \pi^{2}-\left(\frac{1}{2}+\varsigma_{P}\right) \Phi\right]-\Omega^{2}\right\} ; \quad b_{34}=-\frac{8}{3} \Phi ; \quad b_{44}=2 \pi^{2}\left[4 \pi^{2}-\left(\frac{1}{2}+\varsigma_{P}\right) \Phi\right]-\frac{\Omega^{2}}{2} \\
& \varsigma_{A}=\frac{m_{A}}{m_{s h}} ; \varsigma_{P}=\frac{m_{P}}{m_{s h}} ; \varsigma_{B}=\frac{m_{B}}{m_{s h}} ; \quad \Omega^{2}=\omega^{2} \frac{m_{s h} l_{a}^{3}}{\varepsilon} ; \quad \Phi=\frac{-\xi_{A}^{\prime \prime} m_{s h} l_{a}^{2}}{\varepsilon} ; \quad v=\frac{c_{B} l_{a}^{3}}{\varepsilon}
\end{aligned}
$$

are non-dimensional values.

To determine an optimal (as to simplicity and acceptable for engineering accuracy) shape of vibration form of the arrow let's consider reductions of the hypothetic form Eq. (11). One of them describe free motion of the arrow as a rigid shift:

$$
\eta_{a 1}=\eta_{A}+\psi z
$$

and another - as an elastic shift with two nodes:

$$
\eta_{a 2}=\eta_{A}+\psi z+f_{2} \sin \frac{\pi z}{l_{a}} \text {. }
$$

The arrow shows instability when non-dimensional values of the longitudinal acceleration are $\Phi_{1}=14.79$ and $\Phi_{2}=$ 56.47 (the exact solution [19]). An approximate solution based on the hypothetical form Eq. (16) is $\Phi_{1}=15.42$ (error 4.3\%), and on the hypothetical form Eq. (11) is $\Phi_{1}=14.56$ (1.6\%) and $\Phi_{2}=65.68(16.3 \%)$. The hypothetical form Eq. (15) doesn't cover instability with the longitudinal acceleration $\Phi_{1}$ and $\Phi_{2}$, and the form Eq. (16) describes instability only with one value of the longitudinal acceleration $\left(\Phi_{1}\right)$. 
Table 1

Values of natural frequencies for different models of the bow and arrow oscillation forms (an error relatively the exact solution [19])

\begin{tabular}{cccc}
\hline Form & \multicolumn{3}{c}{$\Phi$-non-dimension acceleration parameter } \\
\cline { 2 - 4 } model & 0 & 10 & 20 \\
\hline$[19]$ & 0 & $5.29 \mathrm{j}$ & $12.41 \mathrm{j}$ \\
$(15)$ & 0 & $4.90 \mathrm{j}(-7.4 \%)$ & $7.55 \mathrm{j}(39 \%)$ \\
$(16)$ & 0 & $5.24 \mathrm{j}(-0.5 \%)$ & $11.46(-7.5 \%)$ \\
$(11)$ & 0 & $5.30 \mathrm{j}(0.2 \%)$ & $12.65(2.0 \%)$ \\
{$[19]$} & 6.63 & 5.43 & $5.83 \mathrm{j}$ \\
$(15)$ & $7.01(5.7 \%)$ & $5.90(9.8 \%)$ & - \\
$(16)$ & $6.65(0.3 \%)$ & $5.52(1.76 \%)$ & $5.30 \mathrm{j}(-9.1 \%)$ \\
$(11)$ & $6.64(0.2 \%)$ & $5.44(0.2 \%)$ & $5.69 \mathrm{j}(-2.4 \%)$ \\
{$[19]$} & 15.66 & 8.63 & 5.46 \\
$(15)$ & - & - & $5.42(-0.7 \%)$ \\
$(16)$ & $15.70(0.3 \%)$ & $8.81(2.1 \%)$ & $5.47(0.2 \%)$ \\
$(11)$ & $15.69(0.2 \%)$ & $8.55(0.9 \%)$ & $5.46(0.0 \%)$ \\
{$[19]$} & 45.96 & 41.16 & 35.80 \\
$(11)$ & $46.01(0.1 \%)$ & $41.25(0.2 \%)$ & $36.17(1.0 \%)$ \\
\hline
\end{tabular}

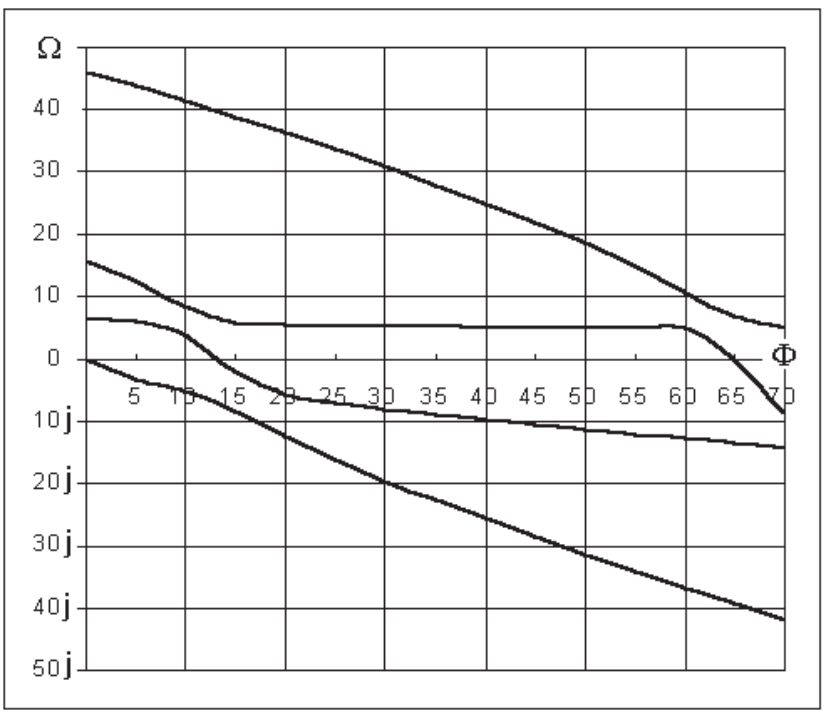

Fig. 3. Natural frequencies vs. longitudinal acceleration of the arrow: $j=(-1)^{1 / 2}$-imaginary unity.

According to the data in Table 1 and graphs (Fig. 3), all three hypothetic forms are accompanied with the exact (atrophied) solution $\left(\Phi_{0}=0\right)$.

Like the previous problem (symmetrical scheme), the solution was obtained using the MathCAD package. So, the method to study vibration processes in the bow and arrow system is realized with standard programs from well-known computer packet.

The graphs show decreasing of natural frequencies values as longitudinal acceleration increases (see Fig. 3). One of the natural modes (while acceleration) presents instability of the arrow, i.e. monotone increase of its angle with the longitudinal axis. As a non-dimensional value of the acceleration equal $\Phi=15$, the instability appeared 'thanks' the first natural mode that has one node, and as $\Phi=65$ - the second mode with two nodes.

In the range of small and average accelerations $(\Phi<10)$, the error of solutions for the natural frequencies as we use hypothetic forms with two (16) or three (11) nodes is appreciate for engineering calculations $(\sim 2 \%)$. In the range of high accelerations $(\Phi>20)$, the same accuracy is possible as we use the hypothetic form with three nodes (see Table 1). As we use the form with one node (15), the error becomes greater than 5\%, that is appreciated only 
for a previous analysis of vibrations when we can ignore the arrow deformation.

\section{Experimental verification}

The results of high-speed video footage [12] show the duration of bow and arrow common motion ranges $18 \div$ $19 \mathrm{~ms}$. During this time, the arrow makes one full circle of vibration with two-node bending form. We can apply data results of our modeling (see Section 4 and Fig. 3) because they are obtained for the most popular modern sport bow designed in the frames of the FITA Standard. There is well-known correlation between bow and arrow parameters (www.archery.org). The arrow, used in the video footage, had a length $l_{a}=712 \mathrm{~mm}$; the mass of the shaft was $m_{s h}=14.3 \mathrm{~g}$; the distributed bending stiffness was $\varepsilon=4.76 \mathrm{Nm}^{2}$. Non-dimensional longitudinal acceleration is $\Phi=5.28$ that provides us with a second (from the top of the Fig. 3) curve line to a non-dimensional natural frequency $\Omega=11.2$ regards to the two-node form. A period of bow and arrow vibration according to this form, which is in good agreement with the video footage, is (see notes to Eq. (14)): $T=\frac{2 \pi l_{a}}{\Omega} \sqrt{\frac{m_{s h} l_{a}}{\varepsilon}} \approx 0.0184$ (s).

\section{Conclusion}

1. Bow and arrow common motion is characterized by intensive dynamic processes, i.e. impulse buckle instability and bend vibrations.

2. An engineering method of calculation of the vibration processes in the system is based on the model of dynamic longitudinal bend and buckle of Euler-Bernoulli shift, with a simple mechanical oscillator connected to its back end.

3. The shape of the hypothetic vibration mode could be constructed with a linear function and harmonics in the form of a sinusoid and a one-half of sinusoid that gives a solution with increased accuracy, as for the engineering calculations ( $\sim 2 \%$ error).

4. The model of bow and arrow vibrations is approbated using the standard programs of the common software package MathCAD, this enables the method to be used in the engineering practice and in the sport of archery.

\section{Acknowledgements}

Dr. J.C. Slater, Wright State University and anonymous referee.

\section{Appendix. Reduction to the symmetrical scheme}

A modern archery sport bow consists of two equal limbs, but the bow and arrow system cannot be a symmetrical relatively the arrow axis in the vertical plane. The hand holding the bow and the arrow cannot settle dawn simultaneously on the same axis of symmetry. Because this very clear reason, the arrow is situated a little above of the hand holding the bow in the middle of the handle. Hence, the bow and arrow system is asymmetric in the vertical plane.

Taking into account a common precision that is acceptable for engineering calculations, lets show why we use an equal symmetrical scheme. Because string elasticity is too small and does not matter in the problem of bow and arrow vibration (see Section 3), we can ignore string strength. A distance between the noke points of the two schemes (symmetrical and asymmetrical) in the relevant neutral position is (see Fig. 1):

$$
\eta_{A 0}= \pm h \pm l \cos \theta_{U / L}^{a s y m} \mp\left(s_{0} \mp \Delta s_{0}\right) \cos \gamma_{U / L}^{a s y m},
$$

where $\Delta s_{0}$ is a half of the difference in the length of the lower and upper branches of the string. The rest nomenclatures have been introduced in the main part of the paper.

Relevant distance from the handle to the noke point is: 


$$
\xi_{A}=l \sin \theta_{\mathrm{U} / \mathrm{L}}^{a s y m}+\left(s_{0} \mp \Delta s_{0}\right) \sin _{\mathrm{U} / \mathrm{L}}^{\text {asym }} .
$$

Like it is in the section, let's connect together geometric equations for the neutral (1) and displaced (A1), (A2) positions of the chain:

$$
\theta_{U / L}^{a s y m}=\theta+\Delta \theta_{U / L}^{a s y m} ; \quad \gamma_{U / L}^{a s y m}=\gamma+\Delta \gamma_{U / L}^{a s y m} .
$$

Since the asymmetry is small $\left(\frac{\Delta s_{0}}{s_{0}} \sim 10^{-1,5}\right)$, we can assume $\sin \chi \approx \chi ; \cos \chi \approx 1$, where $\chi$ is a common definition of the small angles $\Delta \theta_{U / L}^{a s y m}, \Delta \gamma_{U / L}^{a s y m}$. We shall ignore products of small parameters as quantities of a second power of small value. Using the last assumption after substitution (A3) to (A1) and (A2) and some mathematical transformations, we get a linear (regarding the sums in brackets) system of algebraic equations:

$$
\begin{gathered}
l\left(\Delta \theta_{L}^{\text {asym }}+\Delta \theta_{U}^{\text {asym }}\right) \cos \theta+s_{0}\left(\Delta \gamma_{L}^{\text {asym }}+\Delta \gamma_{U}^{\text {asym }}\right) \cos \gamma=0 ; \\
l\left(\Delta \theta_{L}^{\text {asym }}+\Delta \theta_{U}^{\text {asym }}\right) \sin \theta-s_{0}\left(\Delta \gamma_{L}^{\text {asym }}+\Delta \gamma_{U}^{\text {asym }}\right) \sin \gamma=0
\end{gathered}
$$

that has only zero solutions, because its main determinant $(\cos \theta \sin \gamma+\sin \theta \cos \gamma)$ for the real sport bow parameters $(0<\theta+\gamma<\pi)$ has non-zero value: $\cos \theta \sin \gamma+\sin \theta \cos \gamma \equiv \sin (\theta+\gamma) \neq 0$. Therefore we can write:

$$
\begin{gathered}
\Delta \theta_{L}^{\text {asym }}=-\Delta \theta_{U}^{\text {asym }}=\Delta \theta_{\text {asym }} ; \\
\Delta \gamma_{L}^{\text {asym }}=-\Delta \gamma_{U}^{\text {asym }}=\Delta \gamma_{\text {asym }} .
\end{gathered}
$$

Using (A1), (A2), and (A4), we write expressions of velocities for the asymmetrical scheme:

$$
\begin{aligned}
\eta_{A}^{\prime}= & -\sqrt{\xi_{A}^{2}+\eta_{A 0}^{2}} \kappa^{\prime} \mp l\left(\theta^{\prime} \mp \Delta \theta_{\text {asym }}^{\prime}\right) \times \\
& {\left[\sin \theta \mp \Delta \theta_{\text {asym }} \cos \theta-\frac{\left(\cos \theta \pm \Delta \theta_{\text {asym }} \sin \theta\right)\left(\sin \gamma \mp \Delta \gamma_{\text {asym }} \cos \gamma\right)}{\cos \gamma \pm \Delta \gamma_{\text {asym }} \sin \gamma}\right] . }
\end{aligned}
$$

Because small quantities $\left(\Delta \theta_{\text {asym }}<<\theta ; \Delta \theta_{\text {asym }}^{\prime}<<\theta^{\prime} ; \Delta \gamma_{\text {asym }}<<\gamma ; \eta_{A 0}<<\xi_{A}\right)$, the expression are reduced to the form equal to the expression for the symmetrical scheme:

$$
\eta_{A}^{\prime}=\mp l \theta^{\prime}(\sin \theta-\cos \theta t g \gamma)-\xi_{A} \kappa^{\prime} .
$$

\section{References}

[1] P. Bajer, L. Bowers, C.R. Fowkes and S. Schoeh, Instructor's manual. (Ed: R.E. Rowe). - Colorado Springs: National Archery Association of USA, $1982.218 \mathrm{p}$.

[2] H. Gros and I. Zanevskyy, Archer-bow-arrow system adjustment in the vertical plane. Scientific proceeding of the XX International Symposium of Biomechanics in Sports, Ed. K.E.Gianikellis, Universided de Extremadura, Spain, 2002, pp. 469-472.

[3] C.N. Hickman, F. Nagler and P.E. Klopsteg, Archery: the technical side. Redlands: National Field Archery Association, 1947.148 p.

[4] B.W. Kooi and J.A. Sparenberg, On the mechanics of the arrow: Archer's Paradox, Journal of Engineering Mathematics 31 (1997), 285-303.

[5] B.W. Kooi, Archery and mathematical modeling, Journal of the Society of Archer-Antiquaries 34 (1991), 21-29.

[6] B.W. Kooi, On the mechanics of the modern working-recurve bow, Computational Mechanics 8 (1991), 291-304.

[7] W.C. Marlow, Bow and arrow dynamics, American Journal of Physics 49 (1981), 320-333.

[8] S. Ohsima and A. Ohtsuki, Simulation of the shape and dynamics of Japanese bow - Application of large deflection theory. The book of the 4th Int. conf. on the Engineering of Sport, Kyoto, Japan, 2002, 102-107.

[9] Ch. Seay, Column-thrust arrow matching, Archery World 17(5) (1984), 43-48.

[10] T.-C. Soong, An Optimally Designed Archery, Xerox Corp., Rochester, New York, 1986, 16 p.

[11] C. Tuijn and B.W. Kooi, The measurement of arrow velocities and the efficiency of working-recurve bows, European Journal of Physics 13 (1992), 127-134.

[12] Werner Beiter Zeigt. Highspeed film. 1992

[13] I. Zanevskyy, A model of string-limbs stiffness in the lateral plane of the sport bow, in: The Engineering of Sport, (4 ed.), S. Ujihachi and S.J. Haake, eds, Blackwell Publishing, Oxford, UK, 2002, pp. 65-71. 
[14] I. Zanevskyy, Mechanical and mathematical modelling and computer simulation of vibration and impact processes in the "Man and Shooting Device" system, Virtual Non-linear Multibody Systems, NATO Advance Study Institute, Vol. 1, Prague, 2002, $248-252$.

[15] I. Zanevskyy, Ballistics of the sport archery bow and arrow, Technical News 2(19) (2004), 43-47 (in Ukrainian).

[16] I. Zanevskyy, Bow and arrow dynamics in the vertical plane, Scientific proceeding of XXI symposium, Vibrations in Physical Systems, Poznań - Kiekrz, 2004, 4 p.

[17] I. Zanevskyy, Dynamics of "arrow-bow" system, Journal of Automation and Information Sciences 31(3) (1999), 11-17.

[18] I. Zanevskyy, Internal ballistics of the sport bow arrow, Collected Scientific and Technical Articles of Military Institute of State Polytechnic University, Lviv, 2000, No. 1, pp. 24-32 (in Ukrainian).

[19] I. Zanevskyy, Lateral deflection of archery arrows, Sports Engineering 4(1) (2001), 23-42.

[20] I. Zanevskyy, Dynamics of the bow and arrow system in the vertical plane, Machinery 8 (2003), 7-15 (in Ukrainian). 

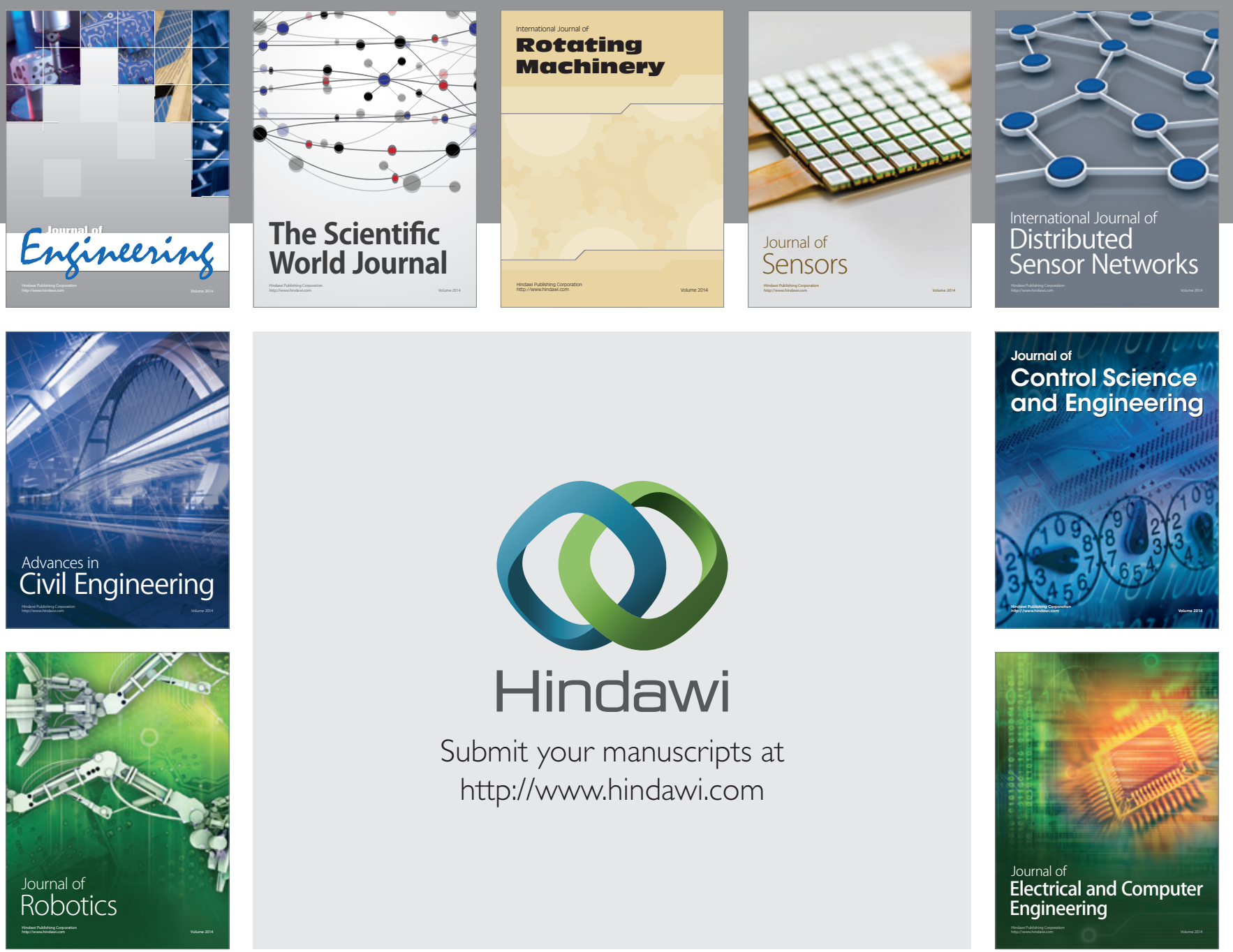

Submit your manuscripts at

http://www.hindawi.com
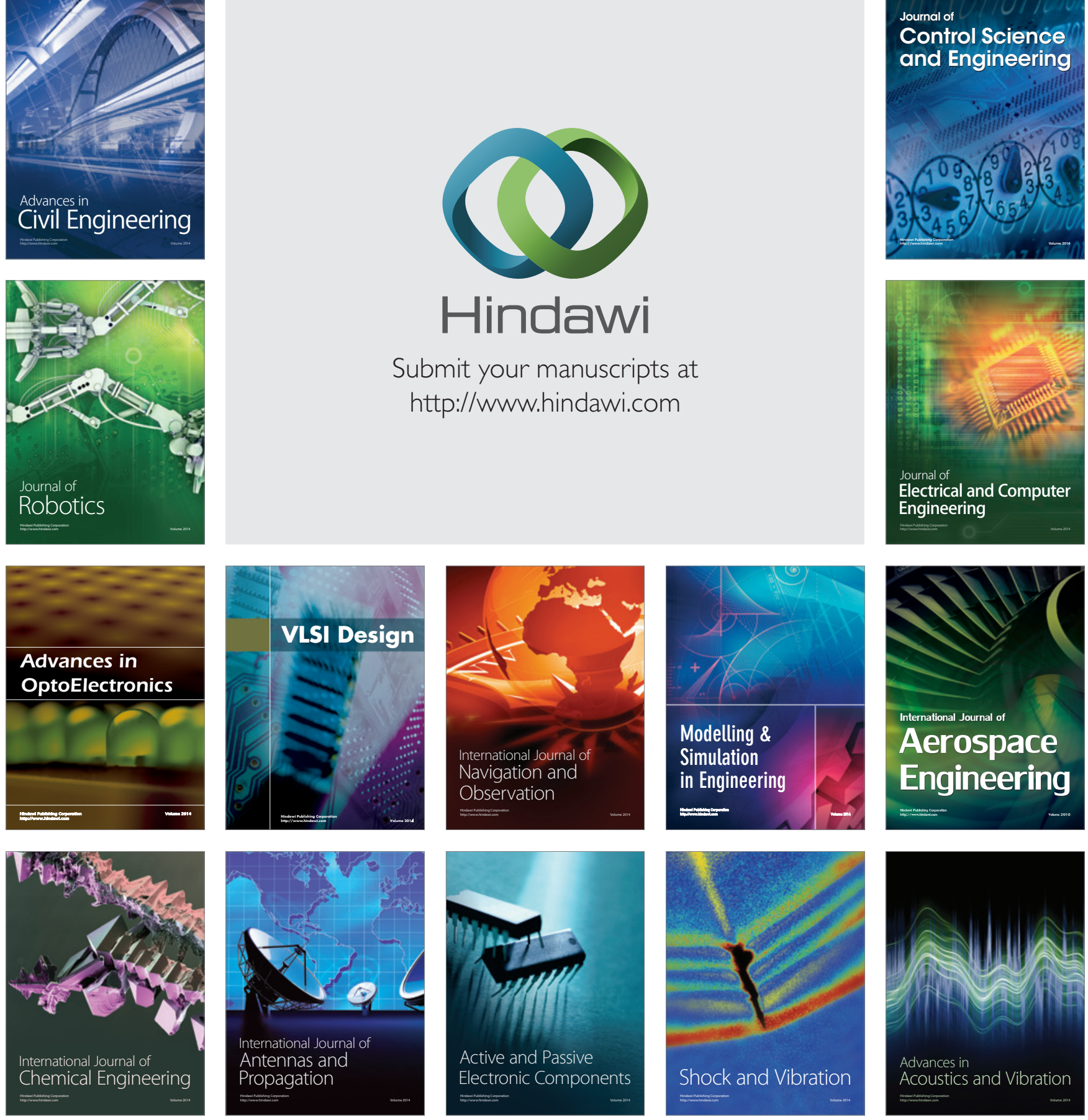